Original Paper http://ajol.info/index.php/ijbcs http://indexmedicus.afro.who.int

\title{
Adventices majeures des parcelles industrielles de canne à sucre des unités agricoles intégrées en Côte d'Ivoire
}

\author{
Oueuga Florence TIALOU ${ }^{1 *}$, Freddy Arnaud YAPI ${ }^{2}$, Konan $\mathrm{KLA}^{1}$ et \\ Kama N'takpé Maxim BORAUD ${ }^{1}$ \\ ${ }^{1}$ Université Félix HOUPHOUËT-BOIGNY, UFR Biosciences, Laboratoire de Botanique 22 BP 582 Abidjan \\ 22, Côte d'Tvoire. \\ ${ }^{2}$ Université Peleforo GON COULIBALY, UFR Sciences Biologiques, Département de Biologie Végétale, BP \\ 1328 Korhogo, Côte d'Tvoire. \\ *Auteur correspondant ;E-mail: jeraude07@gmail.com
}

Received: 02-09-2020 $\quad$ Accepted: 24-03-2021 $\quad$ Published: 30-04-2021

\section{RESUME}

Cette étude vise à identifier les adventices qui constituent la flore des plantations industrielles de canne à sucre des Unités Agricoles Intégrées de Côte d'Ivoire. Elle a permis d'établir la liste des adventices majeures et de montrer leur degré d'infestation dans les exploitations de canne à sucre. Pour mener à bien ce travail, la méthodologie a constitué à faire des placettes de $1500 \mathrm{~m}^{2}(30 \mathrm{~m}$ x $20 \mathrm{~m})$. Dans ces placettes, chaque espèce rencontrée est évaluée par sa présence et son indice d'abondance-dominance. Les familles majoritaires sont celles des Poaceae, des Asteraceae, des Fabaceae, des Euphorbiaceae et des Cyperaceae. La plupart des espèces sont des Dicotylédones avec $69 \%$, dominé par les Thérophytes avec une proportion estimée à $29 \%$. Les espèces d'adventices les plus nuisibles à la culture de la canne à sucre sont : Lactuca taraxacifolia ; Passiflora foetida ; Ipomoea triloba ; Calopogonium mucunoides ; Cyperus rotundus L. ; Cucumis melo L. var. agrestis ; Desmodium scorpiurus; Merremia peltata; Mucuna pruriens; Mondia whitei ; Tridax procumbens; Rottboellia cochinchinensis; Trianthema portulacastrum; Desmodium tortuosum. La connaissance des adventices majeures dans la culture de canne à sucre est la première étape pour la mise en œuvre d'un itinéraire technique efficace de gestion des bioagresseurs.

(C) 2021 International Formulae Group. All rights reserved.

Mots clés : Adventices, Canne à sucre, Unités Agricoles Intégrées.

\section{Major weeds of industrial sugar cane plots of integrated agricultural units in Côte d'Ivoire}

\begin{abstract}
This study aimed at identifying the weeds that make up the flora of industrial sugar cane plantations of the Integrated Agricultural Units of Côte d'Ivoire. It has made to establish the list of major weeds and to show their level of infestation in sugar cane farms. To carry out this work, the methodology used was to make plots of $1500 \mathrm{~m}^{2}$ (30 m x $\left.20 \mathrm{~m}\right)$. In these plots, each species encountered was evaluated by its presence and its
\end{abstract}


abundance-dominance index. The majority families are those of Poaceae, Asteraceae, Fabaceae, Euphorbiaceae and Cyperaceae. Most species are Dicotyledonous with $69 \%$ dominated by Therophytes (29\%). The most harmful adventitious species for the cultivation of sugar cane are: Lactuca taraxacifolia; Passiflora foetida; Ipomoea triloba; Calopogonium mucunoides; Cyperus rotundus L.; Cucumis melo L. var. agrestis; Desmodium scorpiurus; Merremia peltata; Mucuna pruriens; Mondia whitei ; Tridax procumbens; Rottboellia cochinchinensis ; Trianthema portulacastrum ; Desmodium tortuosum. The determination of major weeds in sugarcane cultivation is the preliminary stage of effective fight against biological constraints.

(C) 2021 International Formulae Group. All rights reserved.

Keywords : Weeds, sugarcane, Integrated Agricultural

\section{INTRODUCTION}

La canne à sucre est une graminée tropicale dont la culture est largement répandue avec environ 25 Milliards d'hectares dédiés à la production de cette Poaceae à travers le monde en 2012 (FAO, 2014). C'est une culture de première importance cultivée et répandue à travers le monde car elle présente plusieurs intérêts économiques. Elle est cultivée aussi bien pour le sucre contenu dans ses tiges que pour les coproduits issus de son traitement, comme la production d'électricité, écumes, riches en chaux servant d'amendements pour les parcelles proches de l'usine, ou encore la mélasse et le recyclage rhum ou d'aliments pour le bétail (Morel et al., 2014). Dans l'espace UEMOA, la production sucrière est estimée à près de 400 000 tonnes par année. Malheureusement, cette production ne représente que la moitié des besoins de la population (Morel et al, 2014).

En Côte d'ivoire, il faut au moins 250 000 tonnes de sucre pour satisfaire les besoins de la population (FAO, 2007). Or, selon la FAO (2007), la production sucrière locale est d'environ 200000 tonnes. Cependant, depuis son introduction, les recherches sur la culture de la canne, ont été essentiellement concentrées sur les aspects relatifs à son adaptation. Pourtant, elle est sujette à l'influence de nombreux ennemis qui peuvent provoquer des dégâts souvent importants. Parmi ces ennemis, la part des adventices est moins négligeable et dont l'impact devient plus sensible à l'intensification de la culture. La culture de la canne est sensible à l'enherbement pendant sa phase d'installation (Lebreton, 2010). Des travaux ont montré que dans les pays en voie de développement, la présence des mauvaises herbes peuvent occasionner jusqu'à $25 \%$ de pertes de production dans les zones tropicales et $5 \%$ en zone tempérée (Le Bourgeois \& Marnotte, 2002). De ce fait, la gestion de l'enherbement dans une culture de canne devient importante. Ainsi, est-il nécessaire d'avoir une connaissance précise sur les différentes catégories d'adventices et également sur les facteurs environnementaux responsables de leur développement (Gué, 2017). En Côte d'Ivoire, la quasi-totalité de la production de canne à sucre provient des Unités Agricoles Intégrées de Borotou-Koro et de Zuénoula. La caractérisation de la flore adventice dans les différentes parcelles de ces complexes sucriers serait une solution idoine. Elle permettra la mise au point d'itinéraires techniques efficaces de désherbage qui va garantir de meilleurs rendements et de meilleures productions. C'est dans ce contexte que cette étude intitulée "flore d'adventices majeures des parcelles industrielles de canne à sucre des unités agricoles intégrées en Côte d'Ivoire » a été entreprise. Il s'est agi de caractériser la flore adventice dans la culture de de canne à sucre des Unités Agricoles Intégrées et de dégager les principales espèces problématiques.

\section{MATÉRIEL ET MÉTHODES Présentation du milieu d'étude}

La Côte d'Ivoire compte quatre complexes industriels (Ferké I, Ferké II au Nord, Borotou-Koro et Zuénoula du CentreOuest) qui sont les principales zones de production de canne à sucre et de sucre. Parmi 
ces complexes industriels, deux ont été retenus pour ces travaux de recherche. Il s'agissait des Unités Agricoles Intégrées (U.A.I) de SUCRIVOIRE de Borotou-Koro et de Zuénoula. En plus, elles font partie des principales zones qui, en Côte d'Ivoire, disposent non seulement de grandes superficies de plantations, mais qui ont pour principale activité la culture, l'exploitation, le développement des plantations de la canne à sucre et la production du sucre de canne. Le choix des Unités Agricoles Intégrées de Zuénoula et de Borotou-Koro se justifie par leur situation dans différentes zones climatiques. En effet, l'U.A.I de BorotouKoro appartient à la zone sub-soudanaise avec un climat de type Soudano-Guinéen, tropical humide tandis que celle de Zuénoula se situe dans une zone de transition (Forêt- Savane) qui lui procure un climat de type équatorial et subtropical.

L'Unité Agricole Intégrée (U.A.I) de SUCRIVOIRE de Borotou-Koro est géographiquement localisée entre $8^{\circ} 36^{\prime} 36^{\prime \prime}$ et $8^{\circ} 19^{\prime} 55^{\prime \prime}$ de latitude Nord, et entre $7^{\circ} 9^{\prime} 57^{\prime \prime}$ et $7^{\circ} 9^{\prime} 42^{\prime \prime}$ de longitude Ouest (Figure 1-A), elle couvre une superficie de 9702 hectares (Boraud, 2000). Celle de Zuénoula est située géographiquement entre les coordonnées $7^{\circ} 30$ et $7^{\circ} 40$ de latitude Nord, et entre $6^{\circ} 5$ et $6^{\circ} 15$ de longitude Ouest (Péné et Assa, 2003). Ce complexe s'étend sur une superficie de 11000 hectares dont 6223,22 hectares de plantations industrielles et 723 hectares de cannes à sucre (Figure 1-B).

\section{Matériel d'étude \\ Matériel biologique}

Le matériel utilisé est constitué de l'ensemble des variétés de canne à sucre (Saccharum officinarum) cultivées et des adventices recensées sur les parcelles.

\section{Matériel technique}

Le matériel technique utilisé pour l'inventaire se constitue de :

- carte parcellaire ;

- fiches de relevés ;

- matériel de récolte (sécateur, couteau, ciseau, machette) ;
- papiers journaux pour la conservation des spécimens ;

- appareil photographique numérique pour la prise de vue ;

- matériel informatique constitué d'un tableur Excel 2010 pour la saisie des données ;

- logiciels STATISTICA 7.1 pour la réalisation du diagramme d'infestation.

\section{Méthode d'étude}

La démarche méthodologique s'est faite en deux étapes : la collecte des données et la méthode d'analyse floristique.

\section{Collecte des données}

Une étape préliminaire a été réalisée avant la phase de la collecte des données. Il s'agit du choix du site d'étude. Le choix porté sur les deux Unités Agricoles Intégrées (Zuénoula et de Borotou-koro) se justifie non seulement par le fait qu'elles se situent dans différentes zones climatiques, mais par leur étendue. Ainsi, il offre un avantage de rencontrer un cortège floristique plus important lors de nos relevés floristiques. Pour la collecte de données, la méthode de relevé floristique adoptée est la méthode de relevé itinérant ou « tour de champs ». Cette méthode consiste à parcourir chaque parcelle élémentaire dans toutes les différentes directions possibles jusqu'à la découverte d'une nouvelle espèce. La superficie échantillonnée est de $1500 \mathrm{~m}^{2}$, soit $50 \mathrm{~m}$ de largeur X 20 interlignes de canne X 1,5 m. L'importance relative de chaque adventice a été définie grâce à la note d'abondance dominance attribuée (Tableau 1) au cours des relevés par la méthode de Braun-Blanquet (1932). L'identification des espèces qui n'ont pas pu être identifiées sur le terrain, a été faite à partir de la flore existante au Centre National de Floristique (C.N.F).

\section{Analyse floristique}

L'analyse des données floristique s'est fait en tenant compte de deux approches : une approche qualitative et une quantitative. 
Aspect qualitatif

L'aspect qualitatif de la flore a porté sur le spectre biologique, le type morphologique et le coefficient de similitude de la flore adventices de la canne à sucre dans les parcelles industrielles des unités agricoles intégrées de Borotou-Koro et de Zuénoula.

\section{$>$ Types biologiques}

Les espèces identifiées ont été classées en fonction de leurs types biologiques. Le modèle appliqué dans ce travail est celui de Aké-Assi (1984 et 2002), adapté de celui de Raunkiaer (1905). Pour chaque type biologique, le pourcentage est calculé selon la formule suivante :

$\mathrm{PC}(\mathrm{TB})=\frac{\sum \mathrm{AD} \text { moy des espèces du même }(\mathrm{TB})}{\sum \mathrm{AD} \text { moy de toutes les espèces }}$

$\mathbf{P} \mathbf{C}=$ pourcentage $; \mathbf{T B}=$ Type biologique $;$ AD $\mathbf{m o y}=$ abondance dominance moyenne.

$>$ Types morphologiques

La morphologie végétale est la partie de la botanique qui consiste à décrire la forme et la structure externe des plantes et leurs organes. Ce type de classification tient compte $\mathrm{du}$ port de l'espèce. Il s'agit des espèces arborescentes (arbres et arbustes), des espèces lianescentes et des espèces herbacées (Descoings, 1975).

\section{Coefficient de similitude}

La ressemblance entre les listes des adventices issues de l'inventaire floristique effectué dans les deux types de végétation a été déterminée par la méthode des coefficients de similitude (Cs). Cette méthode des coefficients de similitude est calculée à partir de la formule de Sørensen (1948) qui est la suivante :

$$
\mathrm{Cs}=\frac{2 \mathrm{c}}{\mathrm{a}+\mathrm{b}} \times 100
$$

« $\mathrm{a} »$ et $\ll \mathrm{b} »$ représentent le nombre d'espèces appartenant au deux différentes unités d'échantillonnage ; «c » est le nombre d'espèces communes aux deux listes floristiques.
D'après cette formule, la valeur du coefficient de similitude (Cs) est exprimée en pourcentage. Elle varie entre 0 et $100 \%$. Lorsque le coefficient de similitude est supérieur ou égal à $50 \%$, cela signifie que les deux listes comparées sont très proches l'une de l'autre. En d'autres termes, les deux milieux peuvent être considérés comme floristiquement homogènes (Guinochet, 1973 ; Boraud, 2000). Dans le cas contraire, les milieux comparés sont floristiquement hétérogènes.

Aspect quantitatif

L'aspect quantitatif est relatif à la nuisibilité des adventices. Il tient compte tout d'abord de la fréquence des adventices, ensuite de la détermination de la contribution spécifique des adventices à l'aide d'un calcul et enfin du degré d'infestation par la construction d'un diagramme d'infestation.

$>$ Contribution spécifique due à la fréquence de chaque espèce $\operatorname{Csf}(\mathrm{e})$

C'est l'expression de la contribution qu'une espèce peut apporter à une végétation donnée à partir de sa fréquence absolue. Elle se calcule par la formule proposée par Daget et Poissonet (1969). La contribution spécifique est la fréquence absolue FS(e) de l'espèce (e) sur l'ensemble de la somme des fréquences absolues de toutes les espèces recensées $\sum F S(e)$ que l'on multiplie par cent. En se référant à Daget et Poissonet (1969) et à Aman Kadio (1978) :

- lorsque $\operatorname{Csf}(\mathrm{e})<1 \%$, les adventices sont à effet dépressif plus ou moins négligeable ; pour $1 \%<\operatorname{Csf}(\mathrm{e}) \mathrm{CS}<4 \%$, les adventices sont à effet dépressif élevé ; - quand Csf(e) > $4 \%$, alors les adventices sont à effet dépressif très élevé.

$$
\operatorname{Csf}(e)=\frac{F S(e)}{\sum F S} \times 100
$$

> Potentiel de nuisibilité des adventices

Cette expression traduit l'ampleur des problèmes d'enherbement posés par les différentes espèces d'adventices (Le bourgeois, 1993). La classification des espèces en fonction de leur potentiel de 
nuisibilité est obtenue à partir du diagramme d'infestation. Celui-ci est constitué d'un nuage de points établi à partir des fréquences relatives, en abscisse et des indices d'abondance / dominance moyenne en ordonnée. La fréquence centésimale est le rapport de la fréquence absolue sur le nombre total de relevés multiplié par cent.

La fréquence centésimale $(\mathrm{Fc})$ est la fréquence relative exprimée en pourcentage.

$$
\mathbf{F c}=\frac{\mathrm{Fa}(\mathrm{e})}{\mathrm{N}} \times 100
$$

L'abondance-dominance moyenne de l'espèce (e) est la somme des notes d'abondance dominance sur le nombre total de relevés $(\mathrm{N})$.

$$
\operatorname{AD~moy}(\mathbf{e})=\frac{\sum \text { AD de l' espèce }(e)}{N}
$$

Le Bourgeois (1993) fait une classification qui traduit le potentiel de nuisibilité des différents groupes d'espèces (Tableau 2).

Tableau 1 : Indice d'abondance-dominance Braun-Blanquet (1932).

\begin{tabular}{lc}
\hline Indices & Signification \\
\hline 1 & très rares, rares ou assez abondants, mais recouvrement faible \\
\hline 2 & Individus très abondant ou recouvrement supérieur à $1 / 20$ de la surface \\
\hline 3 & Individus recouvrant $1 / 4$ à $1 / 2$ de la surface, abondance quelconque \\
\hline 4 & Individus recouvrant $1 / 2$ à $3 / 4$ de la surface, abondance quelconque \\
\hline 5 & Individus recouvrant plus de $3 / 4$ de la surface, abondance quelconque \\
\hline
\end{tabular}

Tableau 2: Groupes de mauvaises herbes en fonction de leur fréquence relative et de leur abondance / dominance moyenne (Le Bourgeois, 1993).

\begin{tabular}{llcc}
\hline Indices & Groupes d'adventices & Fréquence relative (Fq Rel) & $\begin{array}{l}\text { Abondance/Dominance } \\
\text { moyenne (A/D moy) }\end{array}$ \\
\hline G1 & $\begin{array}{l}\text { mauvaises herbes majeures } \\
\text { générales }\end{array}$ & $>50 \%$ & $>1,5$ \\
\hline G2 & $\begin{array}{l}\text { mauvaises herbes potentielles } \\
\text { générales }\end{array}$ & $>50 \%$ & $1,25<\mathrm{A} / \mathrm{D}$ moy $<1,5$ \\
\hline G3 & mauvaises herbes générales & $>50 \%$ & $<1,25$ \\
\hline G4 & $\begin{array}{l}\text { mauvaises herbes majeures } \\
\text { régionales }\end{array}$ & $20 \%<\mathrm{Fq}$ rel $<50 \%$ & $1,25<\mathrm{A} / \mathrm{D}$ moy $<1,5$ \\
\hline G5 & $\begin{array}{l}\text { mauvaises herbes potentielles } \\
\text { régionales }\end{array}$ & $20 \%<\mathrm{Fq}$ rel $<50 \%$ & $<1,25$ \\
\hline G6 & mauvaises herbes régionales & $20 \%<\mathrm{Fq}$ rel $<50 \%$ & $>1,5$ \\
\hline G7 & $\begin{array}{l}\text { mauvaises herbes majeures } \\
\text { locales }\end{array}$ & $<20 \%$ & $1,25<\mathrm{A} / \mathrm{D}$ moy $<1,5$ \\
\hline G8 & $\begin{array}{l}\text { mauvaises herbes potentielles } \\
\text { locales }\end{array}$ & $<20 \%$ & $<1,25$ \\
\hline G9 & mauvaises herbes mineures & $<20 \%$ & $<$ \\
\hline
\end{tabular}


O. F. TIALOU et al. / Int. J. Biol. Chem. Sci. 15(2): 594-608, 2021

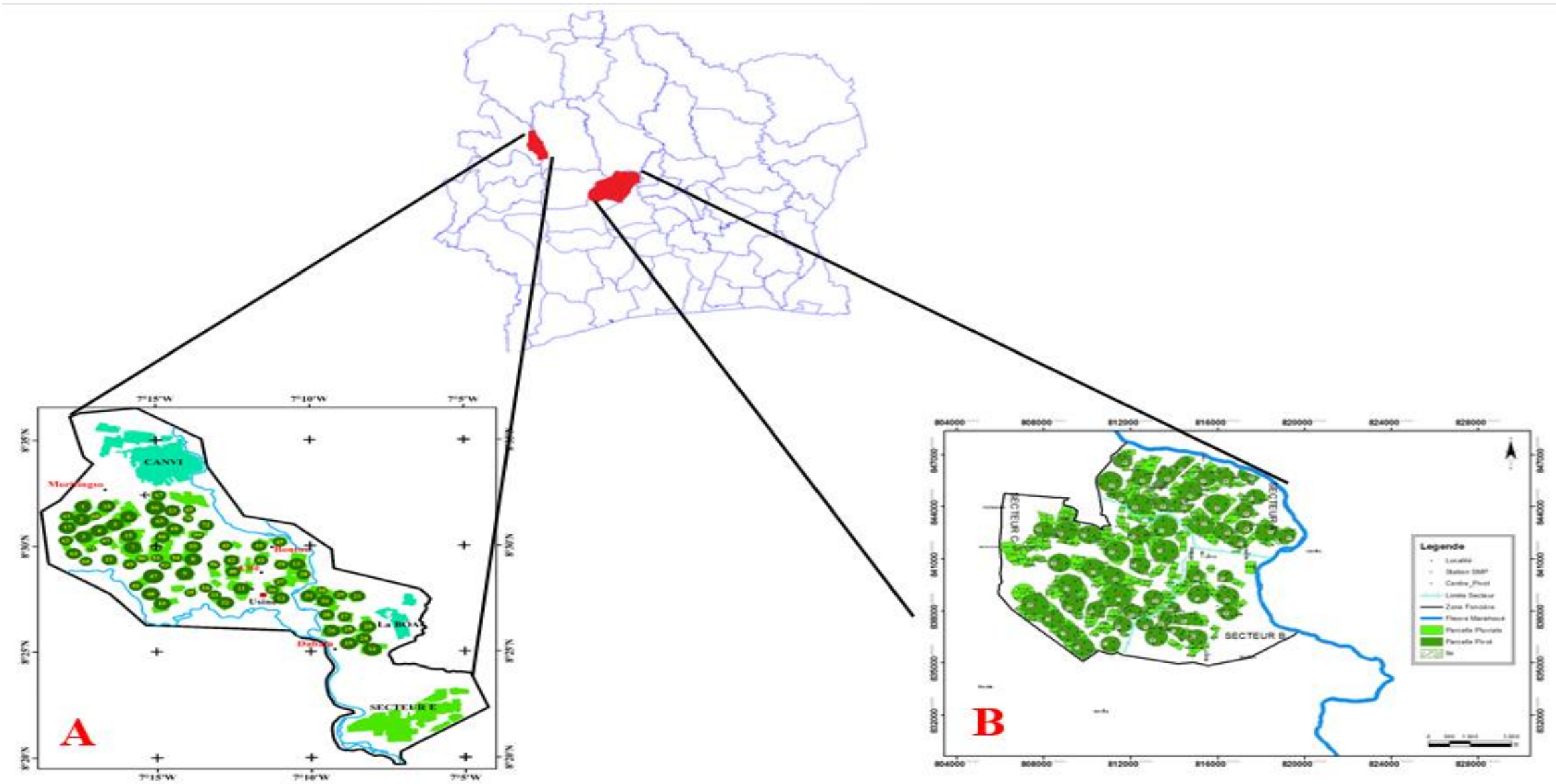

Figure 1 : Localisation de la zone d'étude : Carte parcellaire de l'UAI de Borotou-Koro (A) ; Carte parcellaire de l'UAI de Zuénoula (B) (Carte modifié par TIALOU, 2017). 


\section{RÉSULTATS}

\section{Flore des adventices de la canne à sucre}

L'inventaire floristique réalisé dans les parcelles de canne à sucre des Unités Agricoles Intégrées a permis de recenser 175 espèces reparties entre 120 genres et 40 familles botaniques. Dans la localité de Zuénoula, ce sont 143 espèces adventices reparties entre 102 genres regroupés dans 33 familles botaniques qui ont été recensées. Dans celles de Borotou-Koro, ce sont 149 espèces adventices qui ont été recensées. Ces espèces sont réparties entre 106 genres regroupés dans 38 familles botaniques (Tableau 3). La flore adventice de notre milieu d'étude est dominée par la Classe des Dicotylédones avec $69 \%$ contre $31 \%$ pour les Monocotylédones. Concernant les familles botaniques, la flore adventice de l'ensemble du milieu d'étude est dominée par les Poaceae (31 espèces), les Fabaceae (25 espèces), les Asteraceae (17 espèces), les Cyperaceae (13 espèces) et des Euphorbiaceae (11 espèces). (Tableau 4).

\section{Types biologiques des adventices de la canne à sucre}

La classification des adventices selon le type biologique s'établit par ordre croissant comme suit (Tableau 5): les Thérophytes avec 29\%; viennent ensuite les Nanophanérophytes avec 27\%; puis les Microphanérophytes et les Hemicryptophytes avec respectivement $16 \%$ et $15 \%$ de la flore adventices recensées. Dans la localité de Zuénoula, les thérophytes prédominent avec une proportion de $29 \%$, ensuite viennent les nanophanérophytes avec une proportion de $27 \%$, suivie des microphanérophytes avec $17 \%$ de la flore adventices recensées. De même, dans la zone de Borotou-koro, les mêmes types biologiques ont été obtenus avec les proportions suivantes : les thérophytes (30\%), les nanophanérophytes $(27 \%)$, les microphanérophytes (16\%).

\section{Types morphologiques}

Les types morphologiques recensés sur l'ensemble des parcelles de canne à sucre sont composés des arbustes (b), des herbes (h) et des lianes (1). Sur l'ensemble des parcelles du milieu d'étude, les proportions suivantes ont été obtenues : $47 \%$ pour les herbes, $29 \%$ pour les arbustes et $23 \%$ pour les lianes. Au niveau de l'U.A.I de Borotou-Koro, les types morphologiques sont composés de $45 \%$ pour les herbes, $28 \%$ pour les arbustes, $26 \%$ pour les lianes. À 1'U.A.I de Zuénoula, les types morphologiques sont composés de $44 \%$ d'herbes, $28 \%$ d'arbustes et $27 \%$ de lianes (Tableau 6). Quelle que soit la localité, les herbacées sont les plus abondantes, ensuite viennent les arbustes, puis les lianes.

\section{Coefficient de similitude}

Le calcul des coefficients de similitude a permis de montrer que les flores adventices en générale des plantations de canne à sucre entre l'U.A.I de Zuénoula et de l'U.A.I de Borotou-Koro est supérieur à 50\% (Tableau 7).

\section{Contribution spécifique}

Sur l'ensemble des 175 espèces d'adventices recensées, 34 espèces apparaissent comme agressives pour les cultures de canne à sucre avec un taux de contribution de 66,97\% Ces adventices sont classées par ordre d'agressivité décroissant comme suit : Ipomoea triloba, Lactuca taraxacifolia, Calopogonium mucunoides, Dioscorea bulbifera, Cyperus rotundus, Passiflora foetida, Desmodium scorpiorus, Merremia peltata, Rottboellia cochinchinensis, Dioscorea hirtiflora, Chromolaena odorata, Ipomoea involucrata, Centrosema pubescens, Cucumis melo, Spigelia anthelmia, Laportea aestuans, Euphorbia heterophylla, Amaranthus viridis, Ipomoea heterotricha, etc. (Tableau 8). Dans les parcelles de canne à sucre de l'U.A.I de Zuénoula, sur les 143 espèces recensées, 34 ont une Csf supérieure à 1 et contribuent à hauteur de $66,51 \%$ de la flore adventices de cultures de canne à sucre. Dans les parcelles de canne à sucre de l'U.A.I de Borotou-Koro, sur les 149 espèces recensées, 33 ont une Csf supérieure à 1 et contribuent à hauteur de $69,65 \%$ de la flore adventice des cultures de canne à sucre (Tableau 8).

\section{Potentiel de nuisibilité}

La combinaison de la fréquence et de l'abondance-dominance a permis de mieux apprécier le degré de nuisibilité des adventices. Le diagramme d'infestation 
obtenu à l'issu des 480 relevés fait ressortir cinq groupes sur les neuf représentés à la Figure 2. Les différents groupes représentés sont: le groupe 1 renfermant les mauvaises herbes majeures générales. Ce groupe est représenté par une seule espèce qui est Ipomoea triloba; le groupe 2 (G2) comprenant les mauvaises herbes potentielles générales, renferme neuf espèces que sont: Passiflora foetida, Lactuca taraxacifolia, Calopogonium mucunoides, Dioscorea bulbifera, Centrosema pubescens, Rottboellia cochinchinensis, Merremia peltata, Cyperus rotundus, Dioscorea hirtiflora. Ce sont des mauvaises herbes ubiquistes dont le degré d'infestation est moindre que celui des représentants du groupe 1. Dans le groupe 3 (G3), on a six espèces. Il s'agit de Momordica charantia, Ipomoea involucrata, Cucumis melo var.agrestis, Chromolaena odorata, Desmodium scorpiurus et Euphorbia heterophylla, qui ont presque toutes une abondance inférieure à 1,25 et une abondance relative supérieure à $50 \%$. Ensuite, dans le groupe 6 (G6), 14 espèces sont identifiées. Enfin, le groupe 9 (G9) comprenant les mauvaises herbes mineures. $\mathrm{Ce}$ groupe constitue près de $82,85 \%$ des espèces et ne sont pas nuisibles à la culture.

Tableau 3 : Répartition taxonomique des adventices en culture de canne à sucre dans l'ensemble de la zone d'étude.

\begin{tabular}{llcc}
\hline Localités & $\begin{array}{c}\text { U.A.I Borotou- } \\
\text { Koro }\end{array}$ & U.A.I Zuénoula & $\begin{array}{c}\text { Ensemble de la zone } \\
\text { d'étude }\end{array}$ \\
\hline Nombre d'espèces & 149 & 143 & 175 \\
\hline Nombre de genres & 106 & 102 & 120 \\
\hline Nombre de familles & 38 & 33 & 40 \\
\hline Dicotylédones & 102 & 102 & 120 \\
\cline { 2 - 4 } & $68 \%$ & $71 \%$ & $69 \%$ \\
\hline Monocotylédones & 47 & 41 & 35 \\
\cline { 2 - 4 } & $32 \%$ & $29 \%$ & $31 \%$ \\
\hline
\end{tabular}

U.A.I : Unité Agricole Intégrée

Tableau 4 : Familles botanique d'adventices prépondérantes dans la zone d'étude.

\begin{tabular}{lccc}
\hline & \multicolumn{3}{c}{ Nombre d'espèces } \\
\cline { 2 - 4 } Familles botaniques & U.A.I Borotou-Koro & U.A.I Zuénoula & Ens des U.A.I \\
\hline Asteraceae & $\mathbf{1 2}$ & $\mathbf{1 7}$ & $\mathbf{1 7}$ \\
Commelinaceae & 4 & 4 & 4 \\
Convolvulaceae & 7 & 7 & 7 \\
Cucurbitaceae & 4 & 3 & 4 \\
Cyperaceae & $\mathbf{1 0}$ & $\mathbf{8}$ & $\mathbf{1 3}$ \\
Dioscoreaceae & 8 & 8 & 8 \\
Euphorbiaceae & $\mathbf{7}$ & $\mathbf{1 0}$ & $\mathbf{1 1}$ \\
Fabaceae & $\mathbf{2 2}$ & $\mathbf{1 9}$ & $\mathbf{2 5}$ \\
Malvaceae & 6 & 5 & 6 \\
Poaceae & $\mathbf{2 6}$ & $\mathbf{2 4}$ & $\mathbf{3 1}$ \\
Solanaceae & 5 & 4 & 5 \\
Vitaceae & 3 & 3 & 3 \\
\hline
\end{tabular}


Tableau 5 : Répartition du nombre d'espèces selon les types biologiques dans les U. A. I. de Zuénoula, Borotou-Koro et de l'ensemble de la zone d'étude.

\begin{tabular}{|c|c|c|c|c|c|c|c|c|}
\hline & & \multicolumn{7}{|c|}{ Types biologiques } \\
\hline & & $\overline{\mathbf{C h}}$ & $\bar{G}$ & $\bar{H}$ & $\overline{M p}$ & $\overline{N p}$ & Th & Total \\
\hline \multirow{2}{*}{ U.A.I Borotou-Koro } & Nombre d'espèces & 07 & 14 & 19 & 24 & 40 & 45 & 149 \\
\hline & $\%$ & 05 & 09 & 13 & 16 & 27 & 30 & 100 \\
\hline \multirow{2}{*}{ U.A.I Zuénoula } & Nombre d'espèces & 08 & 12 & 18 & 25 & 39 & 41 & 143 \\
\hline & $\%$ & 06 & 08 & 13 & 17 & 27 & 29 & 100 \\
\hline \multirow{2}{*}{$\begin{array}{l}\text { Ensemble de la zone } \\
\text { d'étude }\end{array}$} & Nombre d'espèces & 08 & 14 & 27 & 28 & 42 & 50 & 175 \\
\hline & $\%$ & 05 & 08 & 15 & 16 & 27 & 30 & 100 \\
\hline
\end{tabular}

U.A.I : Unité Agricole Intégrée ; Ch : chaméphytes ; G : géophytes ; H : hémicryptophytes ; mp : microphanérophytes ; np : nanophanérophytes; Th : thérophytes.

Tableau 6 : Répartition du nombre d'adventices selon les types morphologiques dans les U. A. I. de Zuénoula, Borotou-Koro et de l'ensemble de la zone d'étude.

\begin{tabular}{|c|c|c|c|c|c|}
\hline \multirow{2}{*}{ Localités d'étude } & \multirow{2}{*}{ Nombre d'espèces } & \multicolumn{4}{|c|}{ Types morphologiques } \\
\hline & & Arbres & Herbes & Lianes & Total \\
\hline \multirow{2}{*}{ U.A.I Borotou-Koro } & Nombre d'espèces & 43 & 67 & 39 & 149 \\
\hline & $\%$ & 28 & 45 & 26 & 100 \\
\hline \multirow{2}{*}{ U.A.I Zuénoula } & Nombre d'espèces & 40 & 64 & 38 & 143 \\
\hline & $\%$ & 27 & 45 & 27 & 100 \\
\hline \multirow{2}{*}{$\begin{array}{c}\text { Ensemble de la zone } \\
\text { d'étude }\end{array}$} & Nombre d'espèces & 53 & 83 & 41 & 175 \\
\hline & $\%$ & 29 & 47 & 23 & 100 \\
\hline
\end{tabular}

Tableau 7 : Coefficient de similitude des flores adventices en générale entre les U.A.I de Zuénoula et de Borotou-Koro.

\begin{tabular}{lcc}
\hline & Borotou-Koro & Zuénoula \\
\hline Nombre d'espèces & 149 & 143 \\
\hline Nombre d'espèces communes & \multicolumn{2}{c}{117} \\
\hline Cs $(\%)$ & \multicolumn{3}{c}{80,13} \\
\hline
\end{tabular}


Tableau 8 : Classement des adventices en culture cannière les U. A. I. de Zuénoula, Borotou-Koro et de l'ensemble de la zone d'étude ayant une contribution spécifique $\geq 1$.

\begin{tabular}{|c|c|c|c|}
\hline \multirow[b]{2}{*}{ Espèces } & \multicolumn{3}{|c|}{ Contribution spécifique (\%) } \\
\hline & Borotou & Zuénoula & Zone d'étude \\
\hline Ipomoea triloba $\mathrm{L}$. & 4,96 & 4,36 & 4,65 \\
\hline Dioscorea bulbifera $\mathrm{L}$. & 4,60 & 2,47 & 3,49 \\
\hline Calopogonium mucunoides Desv. & 3,95 & 3,17 & 3,54 \\
\hline Dioscorea hirtiflora Benth. & 3,93 & 1,04 & 2,42 \\
\hline Lactuca taraxacifolia Forssk. & 3,53 & 4,07 & 3,81 \\
\hline Chromolaena odorata (L.) R.King \& H.Robyns. & 3,36 & 1,45 & 2,36 \\
\hline Cyperus rotundus $\mathrm{L}$. & 3,00 & 3,07 & 3,04 \\
\hline Desmodium scorpiurus (Sw.) Desv. & 2,77 & 2,26 & 2,50 \\
\hline Ipomoea involucrata P.Beauv. & 2,56 & 1,99 & 2,26 \\
\hline Passiflora foetida $\mathrm{L}$. & 2,44 & 3,49 & 2,99 \\
\hline Merremia peltata $\mathrm{L}$. & 2,23 & 2,72 & 2,48 \\
\hline Spigelia anthelmia $\mathrm{L}$. & 2,08 & 1,91 & 1,99 \\
\hline Laportea aestuans (L.) Chew & 2,06 & 1,80 & 1,92 \\
\hline Centrosema pubescens Benth. & 2,00 & 2,47 & 2,24 \\
\hline Euphorbia heterophylla L. & 1,81 & 1,80 & 1,80 \\
\hline Ipomoea heterotricha $\mathrm{L}$. & 1,81 & 1,22 & 1,50 \\
\hline Rottboellia cochinchinensis (Lour.) Clayton & 1,74 & 3,13 & 2,46 \\
\hline Cissus adenocaulis $\mathrm{L}$. & 1,47 & - & 1,20 \\
\hline Cucumis melo L. var. agrestis Naud. & 1,47 & 2,55 & 2,03 \\
\hline Amaranthus viridis $\mathrm{L}$. & 1,45 & 1,64 & 1,55 \\
\hline Desmodium triflorum (L.) DC. & 1,45 & - & 1,04 \\
\hline Dioscorea preussii $\mathrm{Pax}$ & 1,45 & - & 1,21 \\
\hline Brachiaria deflexa (Schumach.) Robyns & 1,41 & 1,22 & 1,31 \\
\hline Physalis angulata $\mathrm{L}$. & 1,34 & 1,56 & 1,46 \\
\hline Mondia whitei (Hook.f.) Skeels & 1,32 & 1,47 & 1,40 \\
\hline Tridax procumbens $\mathrm{L}$. & 1,28 & 2,05 & 1,68 \\
\hline Momordica charantia $\mathrm{L}$. & 1,26 & 1,18 & 1,22 \\
\hline Panicum maximum Jacq. & 1,24 & 1,02 & 1,13 \\
\hline Euphorbia hirta L. & 1,22 & 1,66 & 1,45 \\
\hline Mucuna pruriens (L.) DC. & 1,18 & 1,18 & 1,18 \\
\hline Trianthema portulacastrum $\mathrm{L}$. & 1,18 & 1,20 & 1,19 \\
\hline Digitaria horizontalis Willd.+ & 1,11 & 1,18 & 1,15 \\
\hline
\end{tabular}


Coccinia grandis (L.) Voigt

$1,01 \quad 1,62$

Ageratum conyzoides L.

1,06

Dioscorea cayennensis L.

1,08

Eleusine indica (L.) Gaertn.

1,25

Pergularia daemia (Forssk.) Chiov.

1,20

TOTAL

69,64

66,51

66,96

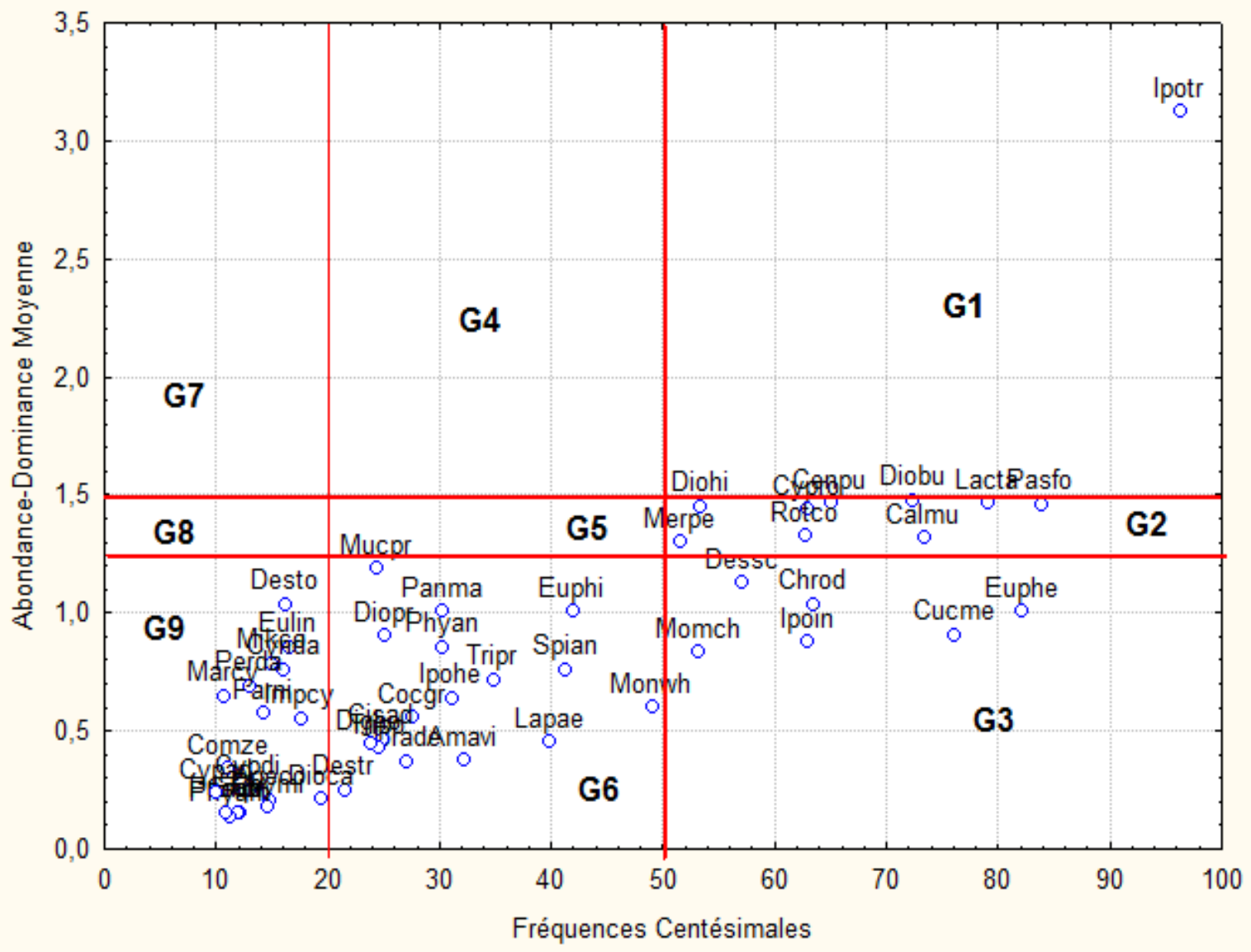

Figure 2 : Diagramme d'infestation des parcelles des Unités Agricoles Intégrées de Côte d'Ivoire. 


\section{DISCUSSION}

L'étude de la flore adventice des parcelles de canne à sucre des Unités Agricoles Intégrées inventoriées montre une grande diversité et richesse spécifique. La flore adventice obtenue dans cette étude est de 175 espèces. Lebreton (2010) a obtenu 155 espèces dans les parcelles de canne à sucre à la Réunion. Nos résultats sont moindres que ceux de Boraud (2000) qui a recensé 234 espèces en culture cannière dans le domaine soudanais et ceux de Ahonon et al (2018) qui quant à lui a obtenu 229 adventices dans la culture de Haricot au Centre-Ouest de la Côte d'Ivoire. Le nombre d'espèces obtenu est largement inférieur à ceux obtenus dans plusieurs travaux réalisés en Côte d'Ivoire, dans d'autres spéculations. En culture d'ananas en basse Côte d'Ivoire, Mangara et al. (2010) ont obtenu 239 espèces. Kouamé (2014) a inventorié 227 espèces en riziculture dans le district de Yamoussoukro. Dans le département de M'Bahiakro au Centre-Est de la Côte d'Ivoire, Kouakou (2016) a listé 299 espèces en culture de maïs. Kouadio et al., (2013) dans les plantations de bananiers à Dabou ont dénombré 281 espèces. En culture vivrier, dans la région de la Mé, Yapi (2017) a obtenu 206 espèces d'adventices. Nos résultats sur la diversité de la flore pourraient s'expliquer par divers arguments. L'un des plus importants est le fait que notre étude a eu lieu en milieu industriel où l'utilisation d'herbicide en permanence et les opérations de sarclage périodique contrôlent le cortège floristique. En effet, dans les systèmes typiquement paysans, les conditions de lutte contre les mauvaises herbes sont rudimentaires.

Les grandes familles des Poaceae,

Fabaceae, Asteraceae, Cyperaceae et Euphorbiaceae représentent les familles botaniques les plus importantes. Nos résultats sont conformes à ceux de Lebreton (2010) qui a classé ces familles parmi les plus représentatives des parcelles de canne en Réunion et ceux de Mangara (2010), en culture d'ananas en Côte d'Ivoire. Ces familles figurent parmi celles recensées par
Boraud (2000), Kouadio et al. (2013) et notamment ceux de Ahonon et al. (2018). La prédominance de ces familles pourrait s'expliquer par leur adaptation à des milieux très différents. Une autre explication est le fait que certaines espèces, notamment celle de la famille des Asteraceae, sont anémochores et peuvent ensemencer rapidement les milieux cultivés (Yapi, 2017).

$\mathrm{Au}$ niveau des types biologiques, nos résultats indiquent que quel que soit le milieu d'étude, à l'exception des hydrophytes $(\mathrm{H})$, des mégaphanérophytes (MP) et des mésophanérophytes, tous les autres types biologiques sont représentés dans cette végétation d'adventices. Cependant, dans des proportions différentes. Les Thérophytes (30\%), suivies des Nanophanérophytes (27\%) sont remarquables. Ces deux types biologiques sont les premiers à se mettre en place dans les parcelles cultivées. La culture de canne à sucre se pratiquant en milieu ouvert, cette ouverture crée un milieu bien éclairé qui favorise le développement des espèces héliophiles notamment les Thérophytes. La proportion élevée des thérophytes pourrait s'expliquer par l'intensification des pratiques culturales des parcelles agricoles. De manière générale, les premiers travaux de préparation des parcelles à cultiver (labours ou sarclages) favorisent le développement des Thérophytes et des Nanophanérophytes (Ipou Ipou, 2005 ; Kouakou, 2016).

La comparaison des différentes listes floristiques par la méthode du coefficient de similitude laisse apparaitre que toutes les valeurs obtenues sur les différents sites sont supérieures à $50 \%$. Ces résultats montrent que la flore adventice de la canne à sucre au niveau de différentes parcelles de chaque site est similaire du point de vue composition. Cette ressemblance pourrait s'expliquer par le fait que, nous sommes en monoculture et les pratiques culturales sont les mêmes. Ensuite, par la proximité géographique des zones d'étude (transition forêt-savane). Ipomoea triloba est la seule espèce appartenant au groupe des mauvaises herbes majeures 
générales. La forte nuisibilité d'Ipomoea triloba dans cette étude pourrait s'expliquer par son mode de reproduction et son cycle par rapport à celui de la culture de la canne à sucre. C'est une espèce annuelle qui produit un grand nombre de graines. Grâce à son mode de multiplication, elle est très abondante dans les exploitations de canne à sucre. En plus, elle a une levée très précoce engendrant ainsi sa forte présence sur toutes les parcelles inventoriées. Les espèces telles que Passiflora foetida, Lactuca taraxacifolia, Calopogonium mucunoides, Dioscorea bulbifera, Centrosema pubescens, Imperata cylindrica, Rottboellia cochinchinensis, Desmodium scorpiurus, Merremia peltata, Ipomoea involucrata appartiennent au groupe 2. Leur degré d'infestation est moindre que celui d'Ipomoea triloba. Cependant, leur grande capacité d'adaptation à l'environnement leur confère un potentiel d'envahissement important dans les parcelles de canne à sucre. La majorité des adventices lianescentes de cette étude sont annuelles et présentent une contrainte majeure pour les industriels du secteur sucrier. Le mode le plus courant reste la multiplication sexuée par les graines. Nos résultats sont cohérents et conformes à ceux de Lebreton (2010) et Caro-canne (2010) en Réunion. La nuisibilité des espèces lianescentes se manifeste par leur période de germination qui est non seulement tardive mais qui s'effectue sur une longue période. Une telle stratégie leur permet d'échapper aux interventions de désherbage normalement réalisées au cours des trois premiers mois suivant après la plantation. Leurs tiges volubiles peuvent ralentir la croissance, voir étouffer les plants de canne. De plus, parmi les modes de dispersion des adventices, le vent, l'eau et l'homme sont les agents de dispersion les plus dangereux car ils peuvent permettre l'infestation des parcelles sur de longues distances (Lososová et al., 2006 ; Sauzeau, 2010). Il est important d'évoquer que l'entretien des parcelles par les machines, le labour, la préparation des sols joue un rôle très important dans la dispersion des graines.

\section{Conclusion}

Les 480 relevés floristiques réalisés dans les parcelles industrielles de cannes à sucre des Unités Agricoles Intégrées ont permis d'inventorier 175 espèces reparties entre 120 genres et 40 familles botaniques. On observe une nette dominance des Dicotylédones sur les Monocotylédones. Dans les plantations de canne à sucre en Côte d'Ivoire, les types biologiques les plus présents sont les thérophytes, suivis des Nanophanérophytes. Les familles des Poaceae, Asteraceae, Fabaceae et Euphorbiaceae sont les plus importantes de cette flore. La comparaison deux à deux des listes floristiques, entre différentes entités, par localités et par segments toposéquentiels a montré que les espèces sont floristiquement homogènes car leur coefficient de similitude est supérieur à $50 \%$. Le diagramme d'infestation montre six groupes. Parmi les mauvaises herbes recensées Ipomoea triloba, Passiflora foetida, Lactuca taraxacifolia, Calopogonium mucunoides, Dioscorea bulbifera, Centrosema pubescens, Imperata cylindrica, Rottboelia cochinchinensis, Desmodium scorpiurus, Merremia peltata, Ipomoea involucrata sont les plus nuisibles contre lesquelles des mesures de lutte doivent être entreprises.

\section{CONFLIT D'INTERETS}

Les auteurs déclarent qu'il n'y a aucun conflit d'intérêts lié à cet article.

\section{CONTRIBUTIONS DES AUTEURS}

L'auteur principal OFT a conduit l'étude, collecté, traité les données et a rédigé l'article. Le co-auteur FAY a contribué au traitement des données, corrigé et finalisé le manuscrit. Les co-auteurs KK et KNMB ont corrigé et finalisé l'article.

\section{REMERCIEMENTS}

Nous remercions les responsables des UAI de SUCRIVOIRE de Borotou-Koro et de Zuénoula qui ont initié le projet. Nos remerciements vont également à l'endroit du cabinet CAREN AGRO et ses collaborateurs 
pour leur soutien au cours de l'étude sur le terrain. Les auteurs tiennent aussi à remercier les référés pour leurs remarques constructives qui ont permis l'amélioration du document.

\section{RÉFÉRENCES}

Ahonon BA, Traoré H, Ipou IJ. 2018 : Mauvaises herbes majeures de la culture de Haricot (Phaseolus vulgaris L.) dans la région du Moronou au Centre-Est de la Côte d'Ivoire, Int. J. Biol. Chem. Sci, 12(1): $\quad 310-321 . \quad$ DOI : http://dx.doi.org/10.4314/ijbcs.v12i1.25.

Aké-Assi L. 1984. Flore de la Côte d'Ivoire. Étude descriptive et biogéographique avec quelques notes ethnobotaniques. Thèse de doctorat d'Etat, Université d'Abidjan, Côte d'Ivoire, 1206 p.

Aké-Assi L. 2002. Flore de la Côte d'Ivoire : catalogue systématique, biogéographie et écologie. Volume I: Mémoire de botanique systématique. Conservatoire et Jardin Botanique de Genève ; Boissiera $58 ; 396 \mathrm{p}$.

Aman Kadio G. 1978. Flore et végétation des adventices dans l'hévéaculture en basse Côte d'Ivoire (Station expérimentale de l'I.R.C.A). Étude écologique : Dynamique et structure. Thèse de spécialité. Faculté des Sciences et Technique Université d'Abidjan, Côte d'Ivoire ; 194 p.

Aman Kadio G. 1978 b. Principaux groupes indicateurs dans la flore des adventices de l'hévéaculture de la station expérimentale de l'I.R.C.A. de la forêt de l'Anguédou (Côte d'Ivoire). Annales Universitaires d'Abidjan, Série E, 11 : $101-120 . \quad$ DOI : https://journals.openedition.org/jda/5452

Braun-Blanquet J. 1932. Plant sociology. The study of plant communities (English translation of "pflanzensoziologie" by G. D. Fuller and H. S. Conard). University of Chicago (USA), $439 \mathrm{p}$.

Boraud NKM. 2000. Étude floristique et phytoécologique des adventices des complexes sucriers de Ferké 1 et 2 de Borotou et Zuénoula, en Côte d'Ivoire.
Thèse doctorat de $3^{\text {ème }}$ cycle de l'Université de Cocody - Abidjan; Côte d'Ivoire, $181 \mathrm{p}$.

Caro Canne, 2010. Le fléau de lianes grimpantes. Caro Canne, 20 : 13-16. DOI : n20-mauvaises-herbes-le-fleaudes-lianes-grimpantes/.

Daget P, Poissonet J. 1969. Analyse phycologique des prairies, Applications agronomiques. Docum. N ${ }^{\circ} 48$ CNRSCEPE ; $67 \mathrm{p}$.

FAO. 2007. Interaction entre agriculture et Forêt $20^{\text {ème }}$ session, Comité de l'agriculture, Rome, 25-28 avril 2007, 4 p.

FAO. 2014. Perspectives agricole de l'OCDE et de la FAO, $1-15 \mathrm{p}$.

Gué A. 2017. Étude des caractéristiques biologiques de quelques adventices majeures des cultures vivrières du centre-ouest de la Côte d'Ivoire. Thèse de Doctorat, UFR Biosciences, Université Felix Houphouët-Boigny, Abidjan, Côte d'Ivoire 165 p.

Guinochet M. 1973. Phytosociologie. Masson : Paris 1- 226 p.

Ipou Ipou J. 2005. Biologie et écologie d'Euphorbia heterophylla L. (Euphorbiaceae) en culture cotonnière, au Nord de la Côte d'Ivoire. Thèse de Doctorat de l'Université de CocodyAbidjan ; Côte d'Ivoire, $191 \mathrm{p}$.

Kouakou N. 2016. Étude floristique des adventices et effets de Rottboellia cochinchinensis (Loureio) W. Clayton sur les caractères agronomiques du maïs, Zea mays L. (Poaceae) en culture dans le département de M'bahiakro (Centre-Est de la Côte d'Ivoire). Thèse de Doctorat, Université Felix Houphouët-Boigny, Abidjan, Côte d'Ivoire, $137 \mathrm{p}$.

Kouamé KF. 2014. Contraintes liées à la production rizicole : étude de la flore et de la végétation adventices de la riziculture dans la région du Bélier (Centre de la Côte d'Ivoire). Thèse de Doctorat, Université Felix HouphouëtBoigny Abidjan, Côte d'Ivoire, 137 p. 
Kouadio PY, Tiébré MS, Kassi J, N'Guessan EK. 2013. Diversité floristique et déterminants de l'enherbement des bananeraies industrielles de Dabou au Sud de la Côte d'Ivoire. Journal of Applied Biosciences, 68: 5404 - 5416. DOI :

https:// www.ajol.info/index.php/jab/arti cle/view/95066.

Le Bourgeois T.1993. Les mauvaises herbes dans la rotation cotonnière au Nord Cameroun (Afrique). Thèse de Doctorat, Université de Montpellier II Sciences et Techniques du Languedoc, Montpellier, France, $249 \mathrm{p}$.

Le Bourgeois T, Marnotte P. 2002. La lutte contre les mauvaises herbes. MEMENTO de l'agronome. G. Cirad, MAE : 663 - 684.

Lebreton G. 2010. Effet de l'époque de coupe de la canne à sucre sur l'enherbement et le cycle biologique des Adventices à la Réunion, Mémoire de l'Ecole Pratique des Hautes Etudes, Cirad, (2010) 8-87 p.

Lososová Z, Chytry M, Kühn I, Hàjek O, HorakovaV, Pysek P, Ichy L. 2006. Patterns of plant traits in annual vegetation of man-made habitats in central Europe. Perspectives in Plant Ecology, Evolution and Systematics, 8: 69-81.

DOI: https://www.sciencedirect.com/science/a rticle/abs/pii/S143383196000060.

Mangara A. 2010. Les adventices en culture d'ananas : Ananas comosus. (L) Merr. (Bromeliaceae), dans les localités d'Anguédédou, de Bonoua et de N'douci, en basse Côte d'Ivoire : inventaire et essai de lutte. Thèse de Doctorat de l'Université de CocodyAbidjan. Spécialité : Ecologie Végétale, Option. Malherbologie. 208 p.

Mangara A. N'Da AAA, Traoré K, Kéhé M, Soro K, Touré M. 2010. Étude phytoécologique des adventices en culture d'ananas (Ananas comosus (L.) Merr.) dans les localités de Bonoua et N'douci en Basse Côte d'Ivoire. Journal of Applied Biosciences, 36: 2367-2382. DOI:

www.m.elewa.org/JABS/2010/36/6.pdf.

Morel J, Todoroff P, Bégué A, Bury A, Martiné JF, Petit M. 2014. Toward a satellite-based system of sugarcane yield estimation and forecasting in smallholder farming conditions: A case study on Reunion Island. Remote Sensing, 6(7): $6620 \quad$ - $6635 . \quad$ DOI: https://www.mdpi.com/20724292/6/7/6620.

Péné CB, Assa DA. 2003. Variations interannuelles de la pluviométrie et de 1 'alimentation hydrique de la canne à sucre en Côte d 'Ivoire. Science et changements planétaires/Sécheresse, 14(1): 43-52. DOI: http//www.jle.com/fr/revues/sce/edocs/262863/article.phtml.

Raunkiaer CC. 1905. Types biologiques pour la géographie botanique.

Sauzeau B. 2010. Effet de la structure des paysages agricoles sur la flore adventice. Mémoire de Master 2 EFCE, Université de Rennes 1, France, 18 p.

Sørensen T. 1948. A method of establishing groups of amplitude in plant sociology based on similarity of species content. Det Kongelige Danske Videnskabernes Selskabs Kopenhague, Biologiske Skrifter, 5(4): 1-34. DOI: https://ci.nii.ac.jp/naid/10008878962/en/ GoogleScholar.

Yapi AF. 2017. Mauvaises herbes majeures et itinéraires techniques de désherbage des cultures vivrières de la Région de la Mé au sud-est de la Côte d'Ivoire : Cas du bananier plantain et du manioc. Thèse de doctorat, UFR Biosciences, Université Felix Houphouët-Boigny, Côte d'Ivoire, $143 \mathrm{p}$. 